The rapid development of explicit gaze judgment ability at 3 years

Martin J. Doherty, James R. Anderson, \& Lynne Howieson

University of Stirling

Running head: Children's explicit gaze judgment

The research reported here was supported by the Economic and Social Research

Council, United Kingdom (Award Number: R 000222513).

Address for correspondence:

Martin J. Doherty,

Department of Psychology,

University of Stirling,

Stirling FK9 4LA

Scotland.

Email: m.j.doherty@stir.ac.uk 


\title{
The rapid development of explicit gaze judgment ability at 3 years
}

\begin{abstract}
Two studies examined development of the ability to judge what another person is looking at. In Study 1, 54 2- to 4-year-olds judged where someone was looking in real-life, photograph, and drawing formats. A minority of 2-year-olds but a majority of older children passed all tasks, suggesting the ability arises around 3 years. Study 2 examined the fine-grained gaze judgment of 763 - to 6-year-olds and 15 adults, using gaze differences of $10^{\circ}$ and $15^{\circ}$. Development of gaze judgment was gradual, from chance at 3 years to near-adult level performance at 6 years. Although performance was better when a congruent head turn was included, 3-year-olds were still at chance on $10^{\circ}$ head-turn trials. The findings suggest that the ability to explicitly judge gaze is novel at 3 years and develops slowly thereafter. It therefore does not develop out of earlier gaze-following. General implications for the evolution and development of gaze processing are discussed.
\end{abstract}


The rapid development of explicit gaze judgment ability at 3 years

\section{INTRODUCTION}

The ability to respond to others' attention is present from infancy. Gaze following has been the most intensely researched issue in this area. Infants develop sophisticated use of others' gaze to direct their own attention. Research suggests that by the age of about 18 months children represent gaze as a relationship between a person and an object. Although there is disagreement about the age at which children properly understand this relationship, it is often assumed that their understanding is adult-like by the end of infancy. Here we present data that challenge this assumption, and conclude that it is incorrect. Children have a good working concept of attention, adequate to their needs, but it is qualitatively different from the adult concept.

Previous research suggests that before the age of about 3 years children can explicitly judge others' attention from whole body cues and from congruent head-and eye-direction. However, they cannot consistently judge visual attention based solely on eye-direction. As adults, we realize that the direction of the eyes is the most reliable indicator of attention. By contrast, toddlers struggle to use eye-direction to identify what someone is looking at. This strongly suggests they do not understand its significance. In this critical respect, young children's understanding of attention may differ from that of adults. Below, we briefly review studies that examine the emergence of the ability to judge eye-direction, to specify how children's early concept of attention may differ from the later, adult concept. 


\section{Infant Gaze-Following}

Infants can follow adults' head turns from 3 months onwards (Scaife \& Bruner, 1975; D'Entremont, Hains, \& Muir, 1997). By 12 months, infants will follow another's gaze out of their own visual field, so long as their own gaze does not meet attentiongrabbing objects on the way (Butterworth \& Cochran, 1980; Moore, Angelopoulos, \& Bennett, 1997; Corkum \& Moore, 1998). Moore (Corkum \& Moore, 1998; Moore, 1999) argues that infants may not understand others' attention at this age; instead, the other's looking behavior simply acts as a cue predicting the location of an interesting object.

By the end of the first year, however, children appear to represent gaze as a relationship between a viewer and on object. For example, Woodward (2003) habituated infants to an actor looking to one side at an object. Twelve-month-olds dishabituated to the actor looking at a novel object on the same side, but not to the actor looking at the same object on the opposite side. This suggests that they encode a relationship between the actor and object, rather than simple surface features of the events. Younger children showed no preference between the events, and did not dishabituate to either.

There is further evidence that specific properties of the relationship are being represented by 18 months. Butler, Caron, and Brooks (2000) showed that 18-montholds were less likely to follow an adult's head turn to a target when the latter was occluded by an opaque barrier, than when the occluder was transparent. Fourteenmonth-olds also looked less often when there was a screen, but less often still when there was a window. Many 18-month-olds attempted to look around the opaque barrier (see also Dunphy-Lelii \& Wellman, 2004), presumably believing that the adult was looking at an object there; 14-month-olds rarely did this. 
By 18 months, children represent a spatial relationship between object and viewer. They also understand that the relationship is between the viewer and the first object in their line of sight. At around this time children also start to track another's gaze to the space behind them (Butterworth \& Cochran, 1980; Butterworth \& Jarrett, 1991; see Deák, Flom and Pick (2000) for evidence that this ability may emerge earlier). This suggests that children expect gaze to terminate at an object, clear evidence that they represent gaze as a marker of some kind of relationship between a viewer and an object.

The evidence only warrants the conclusion that children represent a relationship; there is no evidence concerning what sort of relationship is represented. Attention is properly understood as a representational mental state. What is attended to influences other mental states such as belief, knowledge and desire, and is a critical feature of perception. It might therefore be a relatively early-developing feature of theory of mind. Some researchers conclude that infants' gaze understanding is evidence of a representational understanding of attention. For example, Butler, Caron, and Brooks (2000) argue that 18-month-old infants understand that looking is referential. They define referentiality as a central property of mental states (i.e., aboutness or Intentionality). Others suggest that sophisticated understanding is present even earlier, and possibly innate. Baron-Cohen (1995) for example, suggests that children have an innate eye-direction detector (the EDD) that becomes active within the first four months of life and that computes relationships between the eyes and the object of attention, interpreted as seeing.

However, more parsimonious interpretations of early gaze processing abilities are available. The relation between gazer and object need not be represented as mentalistic, i.e., involving an understanding of representational mental states. For 
example, children might view gazing at something as similar to touching something. Touch between a person and an object can be understood without reference to psychological states such as attention. Gaze, more spatially separated, is harder to infer, but need not be represented as any more mentalistic. Furthermore, there is evidence that appears inconsistent with the richer interpetation.

\section{Preschool Gaze Judgment}

If gaze following tasks indicated a mentalistic understanding of gaze, children should be able to report where someone is looking. They cannot do this reliably until they are 3 years old (Anderson \& Doherty, 1997; Doherty \& Anderson, 1999). Two-year-olds can explicitly judge gaze direction when a congruent head turn is included, indicating an understanding of the task and test questions. However, when the eyes alone indicate gaze direction even 3-year-olds encounter difficulties.

These findings challenge the consensus, but are perfectly compatible with both recent and older work on gaze judgment. For example, Lee, Eskritt, Symons and Muir (1998) found that 3-year-olds were better than chance at judging what a person in a picture was looking at, but only passed about half the trials (Experiments $1-3$ ). Experiments 4 and 5 showed a person on a TV moving her eyes towards one of three objects and children had to say which one she wanted. Even with the inclusion of movement, 2-year-olds' performance was not above chance, or only exceeded chance with feedback; even then performance of 2- and 3-year-olds was poor ${ }^{1}$.

Masangkay, McCluskey, McIntyre, Sims-Knight, Vaughn, and Flavell (1974) found that most 3-year-olds (7 out of 9) but not 2-year-olds (6 out of 16) consistently identified which of several objects an experimenter was looking at. Baron-Cohen and 
Cross (1992) reported that 3-year-olds judged which of two people was looking at them about $75 \%$ of the time, significantly worse than 4-year-olds. Thayer (1977, replicating Lord, 1974) found that even 6-year-olds were poor at judging whether the experimenter ( $2 \mathrm{~m}$ away) was looking at them (at the bridge of the nose) or at varying angles away from the face.

Thus, studies suggest that children's gaze judgment competence is not reached until around 3 years, yet children are very good at following gaze much younger. There are two possible general explanations for this discrepancy. Children's gazefollowing behavior might indicate adult-like understanding of eye-direction, but in an implicit form that is only later available to explicit report. Some process such as Karmiloff-Smith's (1992) Representational Redescription might account for the development from implicit to explicit gaze understanding. Alternatively, young children might not represent gaze as gaze. Doherty (2006) proposed the existence of two distinct gaze processing systems. Two-year-old children may use their sophisticated gaze following system to redirect their own attention, but whatever understanding this is based on is not yet available for the wider cognitive system to make judgments on. Gaze judgment arises as a novel skill around the age of 3 years, possibly in response to increasing interest in others' mental states. Here, we present new data pertinent to Doherty's (2006) two systems hypothesis.

\section{STUDY 1}

Study 1 assessed gaze judgment abilities in children spanning the age range from 2;0 to $4 ; 11$, using real-life and picture versions of the gaze tasks developed by Doherty and Anderson (1999). Those authors used only schematic drawing stimuli with younger children. Further, we video recorded both the experimenter's and child's eye movements to investigate whether children who could not judge gaze direction might 
nonetheless follow gaze. This highlights an additional concern: as eye-direction tends to produce orienting towards the target, children may perform well at our tasks simply because the target consequently becomes more salient.

This could explain why in Masangkay et al. (1974) nearly 40\% of 2-year-olds correctly judged gaze, whereas in Doherty and Anderson (1999) none did.

Masangkay et al. (1974) used targets above, below and to either side of the child. If the child turned to follow the adult's gaze, the other objects would not be visible, thus making the target particularly salient. We tested this by using two versions of the real-life gaze judgment task: one with objects surrounding the child (the "large-frame" task), and the other with objects midway between the child and experimenter (the "small-frame" task). We predicted better performance on the large-frame task, because the other objects would be practically invisible if children oriented towards the target.

In order to rule out simple orienting as the basis for explicit judgments, we added a novel picture task based on one used by Anderson and Doherty (1997). Photographs or drawings showed two viewers, one of whom was looking at an object. The task was to say which viewer was looking at the object. This task does not allow success through simply orienting towards the object of another's gaze since the task is to select a viewer, not the object. Nevertheless, it requires a judgment of a gaze relationship.

For the real-life and photograph gaze tasks, the viewer gazed $25^{\circ}$ away from the mid-line. Doherty and Anderson (1999) used angles of $15^{\circ}$ and $45^{\circ}$ in a real-life gaze task with 3- to 4-year-old children, and found no difference in performance between the two deviations. An angle of $25^{\circ}$ is fairly natural and comfortable. Furthermore, there is evidence that even at this angle, adults find it difficult to keep 
their heads from moving in the same direction as the eyes, and these movements are perceptible to other adults (Doherty \& Anderson, 2001). This angle was used in the present study as a compromise between perceptibility of the eye-direction cue and the possibility of contaminating it with additional head-direction information. The experimenter concealed her eye movements with a hand-held screen.

\section{Method}

\section{Participants}

Fifty-four children (20 girls) took part in the study. Nineteen children came from a university playgroup, 19 children were recruited through advertising in local nurseries and playgroups, and 16 children came from an urban nursery school. Children comprised three age-groups, as follows:

2-year-olds: 18 children from 2;0 to $3 ; 0$, mean age 2;7, $\mathrm{SD}=3.5$ months.

3-year-olds: 18 children from $3 ; 0$ to $3 ; 11$, mean age $3 ; 6, \mathrm{SD}=3.5$ months 4-year-olds: 18 children from 4;1 to $4 ; 11$, mean age 4;5, SD = 3 months. One additional 2-year-old chose not to complete the procedure.

\section{Design}

There were four types of task. The Looking-where task had three formats: drawing, photograph and real-life. The real-life version had two conditions; objects were either mounted on a large frame around the child, or on a smaller frame between the child and experimenter. The Looking-at-you, Point-direction, and Looking-at-the-ball tasks were administered in both drawing and photograph formats. This yielded nine tasks: real life, four drawing tasks, and four photograph tasks. 
Tasks were presented over three sessions, one session for each format. Order of presentation of the three task formats was counterbalanced. No feedback was given during the tasks.

\section{Materials and Procedure}

All drawings were $18 \times 21 \mathrm{~cm}$. All photographs were $10 \times 15 \mathrm{~cm}$. Example stimuli are shown in Figure 1.

\section{Picture Tasks}

\section{Looking-where}

In the drawing-format Looking-where task (Figure 1a), children were presented with a line drawing of a face with eyes looking up and to the left, at one of four targets (a birthday cake, toy plane, teddy bear and train). On the first trial they were asked to name the objects one by one, and then asked, "Which one is he looking at?". This question was repeated with three more pictures, in which the eyes pointed to the top right, top left, and bottom right.

In the photograph-format Looking-where task (Figure 1b), the procedure and questions were the same, but photographs of a man's head and shoulders were shown, surrounded by four objects (a toy train, a cup, a doll, and a toy dog). His head faced forward and eye-direction was approximately $25^{\circ}$ off midline. The object of gaze was in the sequence top left, bottom right, top right and bottom left in the four photographs.

Figure 1 about here 


\section{Looking-at-you}

In the drawing-format Looking-at-you task (Figure 1c), children were presented with a line drawing of two faces; one gazed towards the viewer and the other had the eyes averted to depict gazing away. Children were asked "Which one is looking at you?". This was repeated with three more pictures. The target face was on the left, right, right, and left, respectively. The incorrect face gazed away from the correct face in half the pictures.

The photograph-format Looking-at-you task was analogous (Figure 1d), except that the first and fourth photograph depicted two women, one looking at the viewer and one looking away. The second and third photographs showed two men. Averted gaze was again $25^{\circ}$ off midline.

\section{Point-direction}

In the drawing-format Point-direction task (Figure 1e), children were presented with a schematic line drawing of a man gazing ahead but pointing to one of four shapes. The child was asked "Which one is Sam pointing at?" The question was repeated with three more pictures. Sam pointed to the bottom-right, top-left, bottom-left, and topright, respectively.

The photograph-format Point-direction task was analogous (Figure 1f), with a man shown pointing to one of four objects (a china frog, a teapot, a cup or a toy dog) surrounding him.

\section{Looking-at-the-ball}


In the drawing-format Looking-at-the-ball task (Figure 1g), children were presented with a picture of two faces, one of which was gazing at a red ball suspended at eye level between them. The other's averted eyes indicated looking away. Children were asked "Which one is looking at the ball?". This was repeated with three more pictures. The target face was on the left, right, right, and left, respectively.

The photograph-format Looking-at-the-ball task was analogous (Figure 1h), except that the first and fourth photographs depicted two women and the second and third photographs depicted two men. Both averted and ball-directed gaze were $25^{\circ}$ off midline.

\section{Real-life Task}

Two rectangular wooden frames were constructed with small platforms at each corner to support target objects. The larger frame allowed the child to sit on a chair with head roughly in the center, with the target objects to either side (the frame used was the same as that used in the photograph-format Point-direction task, depicted in Figure 1, with the child sitting where the pointing man is standing). The experimenter, sitting $2 \mathrm{~m}$ away, moved her eyes through $25^{\circ}$ to look at one of the targets. Her head was at the same height as the child's. For the small frame condition, the frame was positioned between the child and experimenter, $1 \mathrm{~m}$ from each. The two frames were constructed so that the experimenter's eyes had to move through exactly the same angle to look at the targets on each frame. (Dimensions: large frame, upper platforms $166 \mathrm{~cm}$ high, lower platforms 34cm high, $200 \mathrm{~cm}$ wide; small frame, upper platforms $140 \mathrm{~cm}$ high, lower platforms $74 \mathrm{~cm}$ high, $100 \mathrm{~cm}$ wide.)

In the both the large-frame and small-frame conditions, the experimenter first asked children to name the target objects positioned at the four corners: a doll, a china frog, a train and a toy dog. She then made eye-contact with the child before 
concealing her eyes with an A4 sized hand-held cardboard screen. Without moving her head, she looked to the bottom left corner, removed the screen then asked "Which one am I looking at?". Children were free to respond by naming the object or by pointing. Once the child answered, the procedure was repeated again with the top right, top left, and bottom right objects, in that order.

Two video cameras were arranged to videotape the experimenter's and child's faces, and the output was mixed to give a split screen recording.

\section{Scoring and analysis}

Tasks were scored on a pass-fail basis. For the Looking-at-you and Looking-at-theball tasks, which had two alternatives per trial, a pass required correct responses on all four trials $(\mathrm{p}=0.0625)$. For all other tasks, which had four alternatives per trial, the criterion was set at three out of four tasks per trial $(p=0.0508)$. The probability of success by guessing alone is therefore roughly comparable for all tasks.

Videotapes of six of the 4-year-olds' looking behavior in the real-life tasks were lost due to theft, and the performance of one 2-year-old on the large frame task was erased accidentally. The remaining videotapes were scored for whether the child's first look was towards the target looked at by the experimenter. Children sometimes looked away from the experimenter when she hid her eyes, so the child's first look was considered only after the child looked back at the experimenter. All available videotapes were scored by two raters. Of 380 trials scored there were nine cases of disagreement, resulting in kappa coefficients as follows: 2-year-olds, $\mathrm{k}=$ 0.90, 3-year-olds, $\mathrm{k}=0.94$, 4-year-olds, $\mathrm{k}=1.00$. Disagreements were resolved by consensus after reviewing the video. 
Alpha was set at $p=0.05$. Differences between means of the three age groups were analyzed using the Kruskall-Wallis One-way Analysis of Variance, and differences between performances on individual tasks were analyzed using the Binomial test (Siegel \& Castellan, 1988). Correlations were analyzed using special cases of the Pearson Product Moment correlation: the Phi coefficient $\left(\mathrm{r}_{\varphi}\right)$ for two dichotomous variables, and the Point-biserial coefficient for one interval and one dichotomous variable (Hinkle, Wiersma \& Jurs, 1979).

Results

Figure 2 shows performance on the drawing, photograph, and real-life looking-where tasks, and the Point-direction control tasks. Performances on the drawing- and photograph-format Point-direction tasks were virtually identical (binomial task, $\mathrm{p}=$ 0.375). Contrary to the prediction, children performed equally well on both real-life conditions (binomial test, $\mathrm{p}=0.375$ ); 18 children failed the large-frame and 21 children failed the small-frame conditions. Performances on the two real-life conditions were therefore combined and performances on the two Point-direction tasks were combined for clarity of exposition.

Figure 2 about here

Children of all ages performed well on the Point-direction tasks, indicating they were paying attention and understood the general task demands. As expected, only a minority of 2-year-olds, but a majority of 3- and 4-year-olds passed each looking-where task, although the age difference for the drawing-format version only 
approached significance (real-life, $\chi^{2}=16.52, \mathrm{df}=2, \mathrm{p}<0.01$; photograph, $\chi^{2}=7.83$, $\mathrm{p}<0.05$, drawing, $\left.\chi^{2}=4.94, \mathrm{p}=0.085\right)$.

Figure 3 about here

Figure 3 shows performance on the Looking-at-you and Looking-at-the-ball tasks. Again, a minority of 2-year-olds, at least half of 3-year-olds and most 4-yearolds passed each task. (Drawing: Looking-at-you, $\chi^{2}=9.96, \mathrm{df}=2, \mathrm{p}<0.01$; Looking-at-the-ball, $\chi^{2}=9.28, \mathrm{df}=2, \mathrm{p}=0.01$. Photographs: Looking-at-you, $\chi^{2}$ $=11.75, \mathrm{df}=2, \mathrm{p}<0.01 ;$ Looking-at-the-ball, $\left.\chi^{2}=12.37, \mathrm{df}=2, \mathrm{p}<0.01\right)$. Comparing adjacent age-groups, between the 2- and 3-year-old groups there was significant improvement on the photograph Looking-at-you and Looking-at-the-ball tasks (Wilcoxon, $\mathrm{W}=270, \mathrm{p}<0.05, \mathrm{~W}=243, \mathrm{p}<0.01$, respectively) and the drawing Looking-at-you task, $\mathrm{W}=252, \mathrm{p}=0.01$ ) but not the drawing Looking-at-the-ball task ( $\mathrm{W}=306$, n.s.). Between the 3- and 4-year-old groups there were no significant differences, the largest difference being between performances on the drawing Looking-at-the-ball task $(\mathrm{W}=279, \mathrm{p}=0.09)$.

\section{Comparison between tasks}

Performances on the two Point-direction tasks were superior to all other tasks (binomial tests, all $\mathrm{p}$ values $<0.01$ ). Performances on the gaze-direction tasks were generally comparable to each other. The exception concerned the photograph-format Looking-at-you task, on which only $43 \%$ of children reached criterion. This was 
slightly poorer than either real-life condition (small frame, 61\%, large-frame 67\%) and either picture Looking-where task (drawing, 65\%, photograph, 59\%) (binomial test, all $\mathrm{p}$ values $<0.05)$. There were no other significant task differences.

Table 1 shows correlations between age and scores on the gaze tasks (performances on photograph and drawing versions combined for clarity of exposition). Performances on all gaze tasks were substantially and significantly correlated with age and with each other. They were also correlated with performance on the Point-direction task, although because performance was near ceiling on this task, little should be concluded from this. The correlation between the Real-life task and the picture Looking-where tasks was particularly strong, even when age and performance on the Point-direction task was partialled out $(\mathrm{r}=0.58, \mathrm{p}<0.01)$. All other partial correlations were significant $(r=0.39$ or greater, all $\mathrm{p}$ values $<0.01)$.

Table 1 about here

\section{Looking behavior in Real-life tasks.}

Table 2 shows the percentage of trials on which children's first look was to the target, the percentage of trials on which children gave the correct answer ${ }^{2}$, and the relationship between these two variables. For both real-life conditions, the proportion of correct first looks and the proportion of correct responses were very similar, and were all strongly correlated. The only significant difference between looking and response concerned the 4-year-old group on the small frame task (Wilcoxon's Z = $2.45, \mathrm{p}=.014)$, and this resulted from several trials in which children named the correct object but did not look away from the experimenter. 
Table 2 about here

\section{Discussion}

This study shows development of the ability to judge gaze over the preschool period. Only a minority of 2-year-olds, at least half of 3-year-olds and most 4-year-olds were able to make explicit judgments of eye-direction when head-direction was kept constant. This was the case regardless of whether judgments were made of a real person, a person in a photograph or of a drawing. All tasks were significantly correlated even after age was partialled out, and did not differ significantly. The only exception was the photograph-format Looking-at-you task, which proved harder than several of the other tasks. This may partly be due to the complexity of processing two gaze relationships in one photograph. Anderson and Doherty (1997) also found that processing two gaze relationships in photographs was harder than in comparable linedrawing stimuli and single-gaze relationship photographs.

These results suggest that what develops is a general ability to explicitly judge eye-direction, either towards the child or towards an object, irrespective of format. The Looking-at-the-ball tasks are a diagnostic test of this ability, since a general tendency to orient to the target of someone's gaze cannot produce success on them. Performance on these tasks did not differ from performances on the other looking tasks and were in fact correlated with them, suggesting that simple orienting was not responsible for successful judgments on the Looking-where tasks. 
The two conditions of the real-life Looking-where task did not differ in difficulty. Interestingly, the present results on all the Looking-where tasks were comparable to those found by Masangkay et al. (1974). Conceivably, Masangkay et al. and the present study simply happened to have developmentally more advanced samples than Doherty and Anderson (1999).

Our prediction was that the large-frame version would be easier because orienting towards one object would render the other objects invisible. However, given that children were told the nature of the task, shown the objects, and had no reason to expect any novel objects or events during the procedure, it could be argued that the normal motivations for gaze following were absent. This is consistent with the finding that even infants are more likely to follow gaze when targets are distinctive and complex rather than identical and plain (Deák, Flom \& Pick, 2000).

\section{STUDY 2}

The question remains, does the ability to judge eye-direction at 3 years of age develop out of children's gaze following skills? If explicit gaze judgments are based on a well-established implicit ability, children should be relatively proficient shortly after they start to make judgments at all. It is reasonable to assume that in a redescription account, much of the difficulty relates to the redescription process itself, and that the knowledge does not have to be substantially relearned.

An alternative possibility is that explicit gaze judgment is a novel skill. If so, it might take more time to develop. Although this skill seems easy to adults, it is plausibly difficult to learn, requiring processing of eye-direction and head-directions of varying congruence. Gradual improvement of accuracy from close to chance at 3 years to reasonable competence sometime later would support the novel skill hypothesis. 
The literature suggests that preschool children's explicit gaze judgment accuracy may develop slowly. For example, Thayer (1977) found that 6-year-olds were substantially poorer than adults at judging whether an experimenter was looking at them or slightly away. Leekam, Baron-Cohen, Milders, and Brown (1997) used a photo judgment task in which an adult looked at one of three rods, separated by 10 or 20 degrees of visual angle. Only $45 \%$ of typically developing 4-year-olds managed 9 out of 18 correct judgments. In this task head-and eye-directions were mixed. Butterworth and Itakura (2000) presented targets separated by $10^{\circ}$, and found that $4 \frac{1}{2}$ year-olds' ability to identify which one an experimenter was looking at was modest, especially if the head remained directed forward.

In Study 2, we examined the development of gaze accuracy using a procedure similar to the studies just cited. We used a live viewer, since the lack of depth information in photographs makes the target difficult to judge from the angle of gaze. Children were also given Doherty and Anderson's (1999) drawing format Lookingwhere and Point-direction tasks to ensure that they had developed some explicit gaze judgment ability and could follow instructions. We also compared accuracy when head and eyes were congruous and when only the eyes moved to the target, head remaining facing forward. We anticipated higher accuracy, but not ceiling performance, in the former condition.

\section{Method}

\section{Participants}

Participants were children from a private nursery, children from each of the first three classes of a local primary school, and 15 adults. The experimenter was a nursery nurse known to most of the children. Each group had roughly equal numbers of each sex: 
3 year olds: 15 children from 3;3 to 3;10, mean age 3;6, SD 2 months.

4 year olds: 20 children from 4;0 to 4;10, mean age 4;6, SD 3 months.

5 year olds: 20 children from 5;2 to 5;11, mean age 5;6, SD 3.5 months.

6 year olds: 21 children from 6;0 to 6;10, mean age 6;6, SD 3.5 months.

Adults: 15 adults from 18 to 55 years, mean age 30 years, SD 13 years.

\section{Design}

Three-year-olds were tested over two sessions. All other participants received all four tasks in one session. The Point-direction and Looking-where picture tasks were administered in that order at the beginning of the session, followed by four gaze discrimination tasks. Both $10^{\circ}$ and $15^{\circ}$ tasks were presented as a block, each with head-direction tasks or eye-direction task first. This yielded four task orders, used approximately equally within each group.

\section{Materials}

The Point-direction and Looking-where tasks were the drawing versions used in Study 1. The gaze discrimination tasks used a wooden rail, $111 \mathrm{~cm} \times 3.5 \mathrm{~cm}$. The experimenter and child sat opposite each other, $60 \mathrm{~cm}$ apart with their eyes at the same level. The rail was placed midway between them, perpendicular to their line of sight with the midpoint directly in front of them. Six wooden rods, $18 \mathrm{~cm}$ long, were placed upright in indentations along the rail. Indentations were spaced such that when the experimenter fixated on them her eyes moved through $15^{\circ}, 30^{\circ}$, and $45^{\circ}$ to either side for the $15^{\circ}$ condition, or $10^{\circ}, 20^{\circ}$, and $30^{\circ}$ to either side for the $10^{\circ}$ condition. Thus targets were separated from each other and from the midline in steps of $10^{\circ}$ or $15^{\circ}$. The rods were topped by brightly colored plastic animal pencil stoppers (rabbit, pig, sheep, cow, cat and dog). 


\section{Procedure}

Point-direction \& Looking-where

These were as in Study 1.

\section{Gaze discrimination}

The experimenter and child sat with the rail between them, as described above, $30 \mathrm{~cm}$ from the midpoint of the rail. Children were encouraged to examine and name the pencil stopper animals. The experimenter then explained that she was going to look at one of the animals, and the child was to tell her which one it was.

To start a trial, the experimenter made eye contact with the child, then looked to one of the animals. In the eye-direction conditions, her head remained facing the midpoint. In the head-direction conditions, she turned her head and eyes toward the target. While maintaining gaze to the target, she asked, "Which animal am I looking at?". For clarity, children were encouraged to point as well as name the object. The target animals' locations were randomized for each participant. All trials to the left were administered first, then all trials to the right. The four conditions were as follows:

Eye-direction, $10^{\circ}, 12$ trials;

Eye-direction, $15^{\circ}, 12$ trials;

Head-direction, $10^{\circ}, 6$ trials;

Head-direction, $15^{\circ}, 6$ trials.

General encouragement but no trial-specific feedback was given. 


\section{Analysis}

Analyses were conducted on the proportion of trials correct for each age group. Onesample t-tests were used to compare performance with guessing levels, and ANOVA and planned t-tests were used to compare between age groups and conditions. Pearson Product Moment correlations were used to measure association.

\section{Results}

The youngest child $(3 ; 3)$ failed all four of the Point-direction and Looking-where trials. All other children passed all four trials of both tasks. This suggests that even the youngest children understood the request to say what someone is looking at. Figure 4 shows performance on the Eye-direction tasks. Chance performance was two trials out of six for each of the left and right sides. The 3-year-old group was at chance for the $15^{\circ}$ trials on both left and right sides (2.2/6, and 2.0/6, respectively). For the $10^{\circ}$ trials they performed below chance $(1.47 / 6$ and $1.0 / 6$, for left and right sides, respectively), significantly so for the right-side trials $[\mathrm{t}(14)=4.58, \mathrm{p}<.01]$, but not the left. Each older group performed above chance for both $15^{\circ}$ and $10^{\circ}$ trials. The below-chance performance of the 3-year-olds stemmed from occasional responding to the wrong side. Since the right-side trials were always presented second, this may be a form of perseveration. Nevertheless, fewer than $10 \%$ of 3-yearolds' responses and $1 \%$ of older children's responses were to the wrong side.

Figures $4 \& 5$ about here

Figure 5 shows performance on the Head-direction tasks. All age groups performed above chance on the $15^{\circ}$ task, and all but the 3 -year-olds performed above 
chance on the $10^{\circ}$ trials. There were few responses to the wrong side: four 3-yearolds made one wrong-side response on the $10^{\circ}$ right-side trials, and one made another wrong-side response on the $10^{\circ}$ left trials. One 6-year-old made a single wrong-side response on the $10^{\circ}$ left trials.

An ANOVA using the four tasks as a within-subjects variable and age groups as a between-subjects variable revealed a main effect of age, $\mathrm{F}(4,86)=27.8, \mathrm{p}<$ $0.01, \eta_{\mathrm{p}}{ }^{2}=0.564$. The overall proportion of successful trials at each age was as follows: 3 years, 37\%; 4 years, 59\%; 5 years, 61\%; 6 years, 77\%; adults, 92\%. There was also a main effect of task, $\mathrm{F}(1,86)=83.1, \mathrm{p}<0.01, \mathrm{\eta}_{\mathrm{p}}{ }^{2}=0.491$, but no interaction, $F(4,86)=1.22$, n.s., $\eta_{p}{ }^{2}=0.054$. Planned t-tests show that for both Eyedirection and Head-direction tasks, performance on the $15^{\circ}$ condition was better than on the $10^{\circ}$ condition, and performance on either condition of the Head-direction task was superior to that on the corresponding Eye-direction conditions (all $t$ values $>2.6$, $\mathrm{df}=90, \mathrm{p}$ values $<0.01, \mathrm{~d}$ values $>0.54)$.

All four tasks were highly positively intercorrelated: $r=0.74$ for the two eyedirection tasks, and $\mathrm{r}=0.56$ for the two head-direction tasks. These strong relationships were largely independent of age: partial correlations were $r=0.67$, and $r$ $=0.49$, respectively, all $\mathrm{p}$ values $<0.01$. The correlations between the $10^{\circ}$ and $15^{\circ}$ eye-direction tasks and the corresponding head-direction tasks were $\mathrm{r}=0.59$ and $\mathrm{r}=$ 0.52 , respectively (age partialled correlations, $r=0.48$ and $r=0.45$, respectively, all $\mathrm{p}$ values $<0.01)$.

Finally, to test for within-task improvement, we compared performance on the first and second three trials of the eye-direction tasks for left and right sides.

Considering the $15^{\circ}$ trials first, for the left side trials, performance on the first three was poorer than performance on the second three trials: $61 \%$ vs. $75 \%$ correct, $t=3.7$, 
$\mathrm{df}=90, \mathrm{p}<0.01, \mathrm{~d}=0.78$. For the right side, performances on the first and last 3 trials were $53 \%$ and $65 \%$, again a significant difference, $t=3.05, \mathrm{df}=90, \mathrm{p}<0.01, \mathrm{~d}$ $=0.64$. Within groups, the difference was significant for the 4-year-olds on each side, and for the 5-year-olds on the right hand side. ${ }^{3}$

For the $10^{\circ}$ trials, no significant differences were found, with scores ranging between $50 \%$ and $57 \%$ correct.

\section{Discussion}

The main finding of Study 2 is that the ability to make fine judgments of eye-direction develops gradually from chance level at 3 years to approaching adult level at 6 years. Adult performance was good but not perfect. Surprisingly, children's ability to make fine judgments of head-and-eye-direction, although superior to their judgments of eye-direction, was far from perfect and underwent a similar development. Unsurprisingly, performance on $15^{\circ}$ conditions of both tasks was superior to performance on $10^{\circ}$ conditions.

The results give credence to our position that gaze judgment is a novel ability. It begins with the ability to make very coarse judgments of eye-direction, limited to the correct quarter of the visual field. Three-year-olds did this, but could not make finer discriminations; this latter ability developed in a roughly linear fashion over the age range. Adult-like ability was not quite reached by 6 years, and even adults were not perfect: $85 \%$ and $87.5 \%$ correct on the $10^{\circ}$ and $15^{\circ}$ Eye-direction tasks, respectively.

Interestingly, within-task improvement occurred only within left-side and right-side trials, but not across the trials as a whole. There were only three eye positions per condition, so after the first three trials participants had usually seen all possible eye deviations. This means that the target could therefore be identified by 
the relative deviation of the experimenter's eyes, without the need to project a line from the eyes towards the target. Thus, our findings may in fact overestimate children's gaze judgment abilities, although not their rule-abstraction abilities.

\section{GENERAL DISCUSSION}

The main findings of this research are as follows:

1. Children are unable to reliably judge eye-direction until around the age of 3 years. This is the case regardless of whether the stimulus faces are real, photographs, or schematic drawings.

2. Judgment ability improves gradually from chance at 3 years to adult-like levels after 6 years. Judgment of congruent head- and eye-direction is better than judgment of eye-direction, but not perfect.

In relation to these findings we consider the following 3 possibilities: First, development might be gradual; from infancy children become increasingly accurate, and incorporate more cues and more specific cues into their gaze judgment abilities. Second, infant gaze localization abilities may reflect a sophisticated understanding of gaze and attention; however, this understanding is not available for explicit

judgments. Third, preschool gaze judgment is qualitatively different from infant gaze understanding, and is not directly based on it.

\section{Gradual development}

Children's gaze localization ability might be rudimentary in infancy, and improve in terms of the type of cue children can use to determine gaze, and the accuracy which 
they can use these cues. Experiment 1 might demonstrate the achievement of a certain level of accuracy, and Experiment 2 show ongoing development of a skill that began in infancy. However, this seems unlikely given that children can follow eye-direction alone from or before 18 months of age. At the very least, this indicates the ability to detect the correct side gazed to. The Looking-where tasks of Experiment 1 required judgment of the correct side and whether gaze was up or down. It is implausible that this small increase in the specificity of gaze localization requires an additional 18 months to develop. The ability to make an explicit judgment about eye-direction appears to be a novel skill at 3 years.

\section{Gaze understanding becomes explicit}

The second possibility is that infants have a good understanding of eye-direction. This understanding effectively guides their abilities to locate what someone is looking at, but is initially unavailable for explicit judgment. According to this idea, the ability to explicitly judge gaze at 3 years indicates that understanding of eye-direction has become available for explicit report, that existing knowledge has become available to the wider cognitive system. Alternatively, existing knowledge could have been 'redescribed' into a format more readily available as a basis for judgments, perhaps similar to the process of 'Representational Redescription' hypothesized by Karmiloff-Smith (1992).

In either case, the now-explicit knowledge would be based on existing knowledge; substantial relearning should not be necessary. The process by which knowledge becomes available to the wider cognitive system might be expected to take some time. Nevertheless, gaze judgment accuracy should be as high as infant gaze following ability shortly after explicit judgment becomes possible. In the present 
study, accurate gaze judgment undergoes protracted development after it first becomes possible, and is still improving roughly three years later. This should not be the case if it is relying on long-established knowledge. The observed pattern is more consistent with children learning to judge gaze direction anew.

It is worth noting that the accuracy of infants' gaze localization abilities is not known. The measure used in gaze-following experiments is often no more precise than whether the infant looks to the correct side. A handful of studies have used more than one target on the same side, typically separated by $60^{\circ}$. However, the age at which children are able to distinguish between head-and-eye turns of less than $60^{\circ}$ or eye movements of smaller magnitude is unknown. The findings of Study 2 suggest infant gaze following may be of rather limited accuracy.

Gaze judgment is a novel skill at 3 years

On the basis of the present findings, we propose that preschool children's gaze judgments are not directly based on their earlier gaze following abilities. Instead, development appears discontinuous, with eye-direction judgment ability starting from first principles at 3 years. At that age accuracy is low, and it increases gradually thereafter.

This conclusion challenges the claim that infants' understanding of others' visual attention is qualitatively adult-like. This may seem counter-intuitive in the light of their impressive gaze-following abilities and flexible response to others' attentional focus. However, a similar conclusion has been reached in research on chimpanzee gaze following, and on the basis of similar evidence presented here. Chimpanzees show many of the same abilities as 18-month-old infants. They will follow the 
experimenter's gaze to the space behind them, and seem to understand that gaze terminates at barriers (Povinelli \& Eddy, 1996a, 1997). Although they apparently represent a relationship between the experimenter's head and the object of attention, they show little evidence of using this understanding except to direct their own attention and anticipate the location of objects. Povinelli and Eddy (1996b) found that chimpanzees were no more likely to use begging gestures towards an experimenter who could see them than one who could not (because he or she was blindfolded, for example).

Nevertheless, chimpanzees appear to have at least a rudimentary understanding of others' attention. For example, in a competitive food-getting situation, Hare, Call, Agnetta and Tomasello (2000) found that subordinate chimpanzees could take account of what a facing dominant chimpanzee could and could not see. When one piece of food was invisible to the dominant chimpanzee due to the presence of an occluding barrier, the subordinate was more likely to approach that piece than an unoccluded piece. Hare, Call and Tomasello (2001) even found that subordinates could take some account of what another chimpanzee had seen previously.

The overall picture of chimpanzees' and human infants' understanding of others' perception indicates the value of a finer distinction than between understanding and not understanding visual attention (e.g., the "third way hypothesis": Hare, Call \& Tomasello, 2001; Call \& Tomasello, 2005). Like chimpanzees, young children are very good at following eye-direction, but poor at using eye-direction to make judgments (although, perhaps better than chimpanzees, they are quite good at making judgments based on head-direction). 
What causes development from good use of attentional cues to understanding of attention as a representational mental state? Moore (2006) proposes that an important intervening step is to understand gaze as subjective. Once children are able to represent others' attentional cues, they have access to two quite different types of gaze information. The other type of information comes from the first-person experience of seeing. Episodes of joint attention involving other people and objects simultaneously expose children to first-person and third-person manifestations of seeing. This allows children to learn a common way of encoding these two manifestations of seeing. These representations are the basis of the understanding that what can and cannot be seen varies from person to person. Until children understand that visual experience can differ between people, there is no need to postulate representational states intervening between the viewer and the object.

Human children demonstrate an understanding of the subjective nature of visual attention from about 2 years. For example, Moll and Tomasello (2006) examined children's reaction to an adult's apparent inability to find a desired object. Two objects were visible to the child, one of which was clearly occluded from the adult's view. The 24-month-olds handed over the occluded toy more often than expected by chance, whereas 18-month-old children performed below chance. Slightly older children are able to hide an object behind an occluder by moving the object. However, children are unable to hide the object by moving the occluder until they are 3 years old (Flavell et al. 1978), and this ability is associated with their ability to make explicit gaze judgments (McGuigan \& Doherty, 2002).

Two-year-olds are also good at keeping track of what others have experienced. O’Neill (1996) found that when enlisting their parent's help for getting a toy on a high 
shelf, $2 \frac{1}{2}$-year-olds made significantly more gestures towards the object, and named it and its location, if the parent had not witnessed it placed there. This recalls Hare et al.'s chimpanzees' sensitivity to another's past perceptual experience. O'Neill suggests that children may understand people as being globally engaged with or disengaged from an ongoing event, and can consider past engagement in order to predict or influence behavior. From this perspective, children's inability to judge attention on the basis of eye-direction may be because eye-direction is not a good cue to what someone is engaged with. It is transient, constantly changing, and relatively difficult to distinguish. Body direction and posture, and particularly head-direction are more useful cues to engagement than eye-direction.

There remains the issue of why children begin to judge eye-direction at around 3 years of age. Several authors have suggested that understanding gaze is a precursor to understanding of belief (e.g., Gopnik, Slaughter, \& Meltzoff, 1994; Baron-Cohen, 1995; Goméz, 1996). This is plausible, but as discussed, the specific cue of eyedirection may not be a particularly important attentional cue for younger children. What causes the realisation of the importance of eye-direction? Our findings suggest one possibility, that this happens because children start to have a notion that things are in others' minds. The dawning understanding of attention as a representational mental state should cause children to realize that eye gaze is critical in determining the moment-to-moment contents of another's mind. This will then motivate them to learn to discriminate it better. Our results suggest that this begins around 3 years, and learning to discriminate eye-direction takes place over a protracted time course.

The present findings are consistent with the view that human gaze following is based on an evolutionarily older mechanism, but not with the idea that this mechanism represents gaze in psychological terms such as seeing (e.g., as proposed 
by Baron-Cohen, 1995). Some gaze following ability certainly seems to be present early on. Hood, Willen, \& Driver (1998) found that 3-month-old infants would shift their attention in response to a change in eye-direction of an adult presented on a video (but see Farroni, Johnson, Brockbank \& Simion, 2000). Children at this age can distinguish direct and averted gaze (Samuels, 1985; Farroni, Johnson, Brockbank \& Simion, 2000; Vecera \& Johnson, 1995); this ability may even be present shortly after birth in humans (Batki, Baron-Cohen, Wheelwright, Connellan, \& Ahluwalia, 2001; Farroni, Csibra, Simion \& Johnson, 2002) and chimpanzees (MyowaYamakoshi, Tomonoga, Tanaka, \& Matsuzawa, 2003).

Adults appear to use two sources of information for determining gaze direction: the relative luminance of the iris and sclera (see Ando, 2002; Langton, Watt \& Bruce, 2000), and the geometrical arrangement of the eyes (see Ando, 2002; Jenkins \& Langton, 2003). The poor visual acuity of young infants means that at 3 months they probably do not resolve the edges of the eye, iris or pupil (Banks \& Salapatek, 1981). However, they are able to perceive contrast at very low spatial frequencies, and this contrast sensitivity would permit detection of luminance differences between the iris and the sclera from a viewing distance of $50 \mathrm{~cm}$. Thus, infants' earliest gaze following may be based on luminance cues. The luminancebased system is computationally much simpler, at the cost of some accuracy (e.g., in uneven lighting conditions). This suggests that a putative innate gaze processing mechanism might operate on luminance cues.

Adults' gaze judgments might therefore involve two systems, one that is innate, fast and approximate, and another learned, more computationally difficult but more accurate. We suggest that the learned system predominates in adult gaze judgments, and begins to be used at 3 years. Until this age, the luminance system may 
be sufficient for children's requirements. Fine gaze discrimination develops slowly because it is not easy, and until the development of theory of mind, it is not necessary.

\section{REFERENCES}

Anderson, J.R., \& Doherty, M.J. (1997). Preschoolers' perception of other people's looking: Photographs and drawings. Perception, 26, 333-343.

Ando, S. (2002). Luminance-induced shift in the apparent direction of gaze. Perception, $\underline{31}$, 657-674.

Banks, M. S., \& Salapatek, P. (1981). Infant pattern vision: A new approach based on the contrast sensitivity function. Journal of Experimental Child Psychology, $\underline{31}, 1$ 45.

Baron-Cohen, S. (1995). Mindblindness. Cambridge, MA: MIT Press/Bradford. Baron-Cohen, S., \& Cross, P. (1992). Reading the eyes: Evidence for the role of perception in the development of a theory of mind. Mind and Language, $\underline{6}, 173-$ 186.

Batki, A., Baron-Cohen, S., Wheelwright, S., Connellan, J., \& Ahluwalia, J. (2000). Is there an innate gaze module? Evidence from human neonates. Infant Behavior

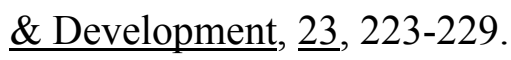

Butler, S.C., Caron, A.J., \& Brooks, R. (2000). Infant understanding of the referential nature of looking. Journal of Cognition and Development, 1 , 359-377.

Butterworth, G., \& Cochran, E. (1980). Towards a mechanism of joint visual attention in human infancy. International Journal of Behavioral Development, $\underline{3}$, $253-272$ 
Butterworth, G., \& Jarrett, N. (1991). What minds have in common is space: spacial mechanisms serving joint visual attention in infancy. British Journal of Developmental Psychology, 9, 55-72.

Butterworth, G., \& Itakura, S. (2000). How the eyes, head and hand serve definite reference. British Journal of Developmental Psychology, 18, 25-50.

Call, J., \& Tomasello, M. (2005). What chimpanzees know about seeing, revisited: an explanation of the third kind. In: N. Eilan, C. Hoerl, T. McCormack, \& J. Roessler (Eds.), Joint Attention: Communication and other minds (pp. 45-64). Oxford: OUP.

Corkum, V., \& Moore, C. (1998). The origins of joint visual attention in infants. Developmental Psychology, 34, 28-38.

Deák, G.O., Flom, R.A., \& Pick, A.D. (2000). Effects of gesture and target on 12and 18-month-olds' joint visual attention to objects in front of or behind them. Developmental Psychology, 36, 511-523.

D'Entremont, B., Hains, S.M.J., \& Muir, D.W. (1999). A demonstration of gaze following in 3- to 6-month-olds. Infant-Behavior-and-Development, 20, 569-572.

Doherty, M.J. (2006). The development of mentalistic gaze understanding. Infant and Child Development, 15, 179-186.

Doherty, M.J., \& Anderson, J.R. (1999) A new look at gaze: preschool children's understanding of eye-direction. Cognitive Development, 14, 549-571.

Farroni, T., Johnson, M.H., Brockbank, M., \& Simion, F. (2000). Infants' use of gaze direction to cue attention: The importance of perceived motion. Visual Cognition, 7, $705-718$. 
Farroni, T., Csibra, G., Simion, F., \& Johnson, M.H. (2002). Eye contact detection in humans from birth. Proceedings of the National Academy of Sciences, 99 , 96029605.

Flavell, J.H., Shipstead, S.G., \& Croft, K. (1978). Young children's knowledge about visual perception: Hiding objects from others. Child Development, $\underline{49}, 1208-1211$.

Goméz, J.C. (1996). Non-human primate theories of (non-human primate) minds: some issues concerning the origins of mind-reading. In: P. Carruthers and P. Smith (Eds.) Theories of theories of mind (pp. 330-343). Cambridge, Cambridge University Press.

Gopnik, A., Slaughter, V., \& Meltzoff, A. (1994). Changing your views: How understanding of visual perception can lead to a new theory of mind. In C. Lewis \& P. Mitchell (Eds.), Children's early understanding of mind (pp.157-181). Hove, England: Lawrence Erlbaum Associates.

Hare, B., Call, J., Agnetta, M., \& Tomasello, M. (2000). Chimpanzees know what conspecifics do and do not see. Animal Behaviour, 59, 771-78.

Hare, B., Call, J., \& Tomasello, M. (2001). Do chimpanzees know what conspecifics know? Animal Behaviour, 61, 139-151.

Hinkle, D.E., Wiersma, W., \& Jurs, S.G. (1979). Applied statistics for the behavioural sciences. Chicago: Rand McNally.

Hood, B.M., Willen, J.D., \& Driver, J. (1998). Adult's eyes trigger shifts of visual attention in human infants. Psychological Science, $\underline{9}, 131-134$.

Jenkins, J., \& Langton, S. R. H. (2003). Configural processing in the perception of eye-gaze direction. Perception, $\underline{32}, 1181-1188$.

Karmiloff-Smith, A. (1992). Beyond modularity: A developmental perspective on cognitive science. Cambridge, MA: MIT Press. 
Langton, S.R.H., Watt, R.J., \& Bruce, V. (2000). Do the eyes have it? Cues to the direction of social attention. Trends in Cognitive Science, $\underline{4}, 50-59$.

Lee, K., Eskritt, M., Symons, L.A., \& Muir, D. (1998). Children's use of triadic eye gaze information for “mind reading”. Developmental Psychology, 34, 525-539.

Leekam, S., Baron-Cohen, S., Perrett, D., Milders, M., \& Brown, S. (1997). Eyedirection detection: A dissociation between geometric and joint attention skills in autism. British Journal of Developmental Psychology, 15, 77-95.

Lord, C. (1974). The perception of eye contact in children and adults. $\underline{\text { Child }}$ Development, $\underline{45}, 1113-1117$.

Masangkay, Z.S., McCluskey, K.A., McIntyre, C.W., Sims-Knight, J., Vaughn, B.E., \& Flavell, J.H. (1974). The early development of inferences about the visual percepts of others. Child Development, $\underline{45}, 349-372$.

Moll, H., \& Tomasello, M. (2006). Level 1 perspective-taking at 24 months of age. British Journal of Developmental Psychology, 24, 603-613.

Moore, C. (1999). Gaze following and the control of attention. In P Rochat (Ed.), Early social cognition: Understanding others in the first months of life (pp. 241256). Mahwah, NJ: Lawrence Erlbaum Associates.

Moore, C. (2006). Commentary on The Development of Mentalistic Gaze Understanding. Understanding the directedness of gaze: three ways of doing it. $\underline{\text { Infant and Child Development, }}$ 15, 191-193.

Moore, C., \& Corkum, V. (1994). Social understanding at the end of the first year of life. Developmental Review, 14, 357-366.

Moore, C., Angelopoulos, M., \& Bennett, P. (1997). the role of movement in the development of joint visual attention. Infant Behavior and Development, 20, 8392. 
Myowa-Yamakoshi, M., Tomonaga, M., Tanaka, M., \& Matsuzawa, T. (2003).

Preference for human direct gaze in infant chimpanzees (Pan trogolodytes).

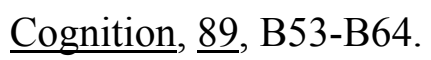

O’Neill, D.K. (1996). Two-year-old children's sensitivity to a parent's knowledge state when making requests. Child Development, $\underline{67}, 659-677$.

Povinelli, D.J., \& Eddy, T.J. (1996a). Chimpanzees: joint visual attention. Psychological Science, 7, 129-135.

Povinelli, D.J., \& Eddy, T.J. (1996b). What young chimpanzees know about seeing. Monographs of the Society for Research in Child Development, $\underline{61}$, no. 3.

Povinelli, D.J., \& Eddy, T.J. (1997). Specificity of gaze-following in young chimpanzees. British Journal of Developmental Psychology, 15, 213-222.

Scaife, M., \& Bruner, J.S. (1975). The capacity for joint visual attention in the infant. Nature, 253, 265-266.

Siegel, S., \& Castellan, N.J. (1988). Nonparametric statistics for the behavioral sciences. Singapore: McGraw-Hill.

Thayer, S. (1977). Children's detection of on-face and off-face gazes. Developmental

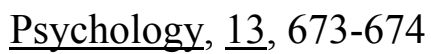

Vecera, S.P., \& Johnson, M.H. (1995). Gaze detection and the cortical processing of faces: Evidence from infants and adults. Visual Cognition, 2 , 59-87.

Woodward, A.L. (2003). Infants' developing understanding of the link between looker and object. Developmental Science, $\underline{6}, 297-311$. 


\section{Footnotes}

1. Furthermore, as well as the added cue of movement, the clown's eyes would have appeared much larger than life. The clown's head and shoulders filled a 32 inch (81 $\mathrm{cm})$ screen, and children were seated 12 inches $(30 \mathrm{~cm})$ away.

2. Note that this differs from the percentage of children passing the task, for which the criterion for success was 3 out of 4 trials correct.

3. When the first trials only are considered, only the 6 year olds were above chance performance, and even then, not for right 15 degrees nor for left 10 degrees. 
Table 1

Correlations between age and tasks of Experiment 1

\begin{tabular}{|c|c|c|c|c|c|}
\hline & Real- Life & $\begin{array}{l}\text { Looking- } \\
\text { where }\end{array}$ & $\begin{array}{l}\text { Looking- } \\
\text { at-you }\end{array}$ & $\begin{array}{l}\text { Looking- } \\
\text { at-the-ball }\end{array}$ & $\begin{array}{l}\text { Point- } \\
\text { direction }\end{array}$ \\
\hline Age & $.47 * *$ & $.43 * *$ & $.48^{* *}$ & $.53 * *$ & .15 \\
\hline Real-life & --- & $.72 * *$ & $.58 * *$ & $.60 * *$ & $.42 * *$ \\
\hline Looking-where & & --- & $.59 * *$ & $.64 * *$ & $.43 * *$ \\
\hline Looking-at-you & & & --- & $.61 * *$ & $.40 * *$ \\
\hline Looking-at-the-ball & & & & --- & $.30 *$ \\
\hline
\end{tabular}




\section{Table 2}

The percentage of trials on which children followed the experimenter's gaze to the target, successful judgment, and their relation

\begin{tabular}{lcccccc} 
Age group & \multicolumn{3}{c}{ Small frame } & \multicolumn{3}{c}{ Large frame } \\
& Looking & Response & Correlation & Looking & Response & Correlation \\
& & & & & \\
& & & & & & \\
2 years & $49 \%$ & $47 \%$ & .64 & $63 \%$ & $56 \%$ & .67 \\
3 years & $85 \%$ & $86 \%$ & .83 & $86 \%$ & $85 \%$ & .83 \\
4 years & $77 \%$ & $90 \%$ & .63 & $85 \%$ & $92 \%$ & .52 \\
\end{tabular}


Figure captions

Figure 1

Example stimuli for the drawing and photograph tasks of Study 1.

Figure 2

Percentage of children reaching criterion on the drawing, photograph and real-life format Looking-where tasks and the Point-direction tasks of Study 1

Figure 3

Percentage of children reaching criterion on the Looking-at-you and Looking-at-theball tasks of Study 1

Figure 4

Percentage of correct judgments in the $10^{\circ}$ and $15^{\circ}$ Eye-direction tasks of Study 2 Figure 5

Percentage of correct judgments in the $10^{\circ}$ and $15^{\circ}$ Head-direction tasks of Study 2 
Figure 1
Click here to download high resolution image

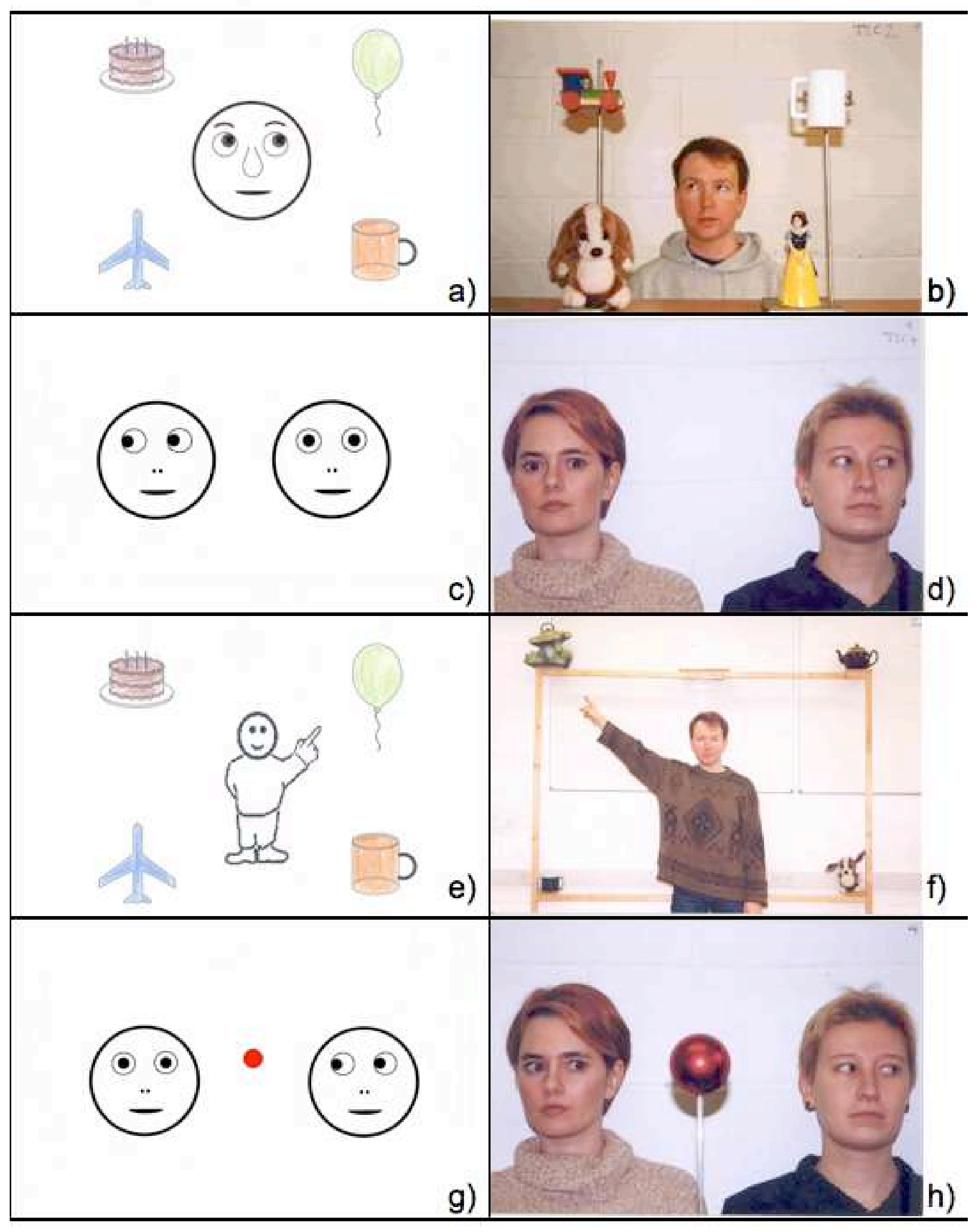




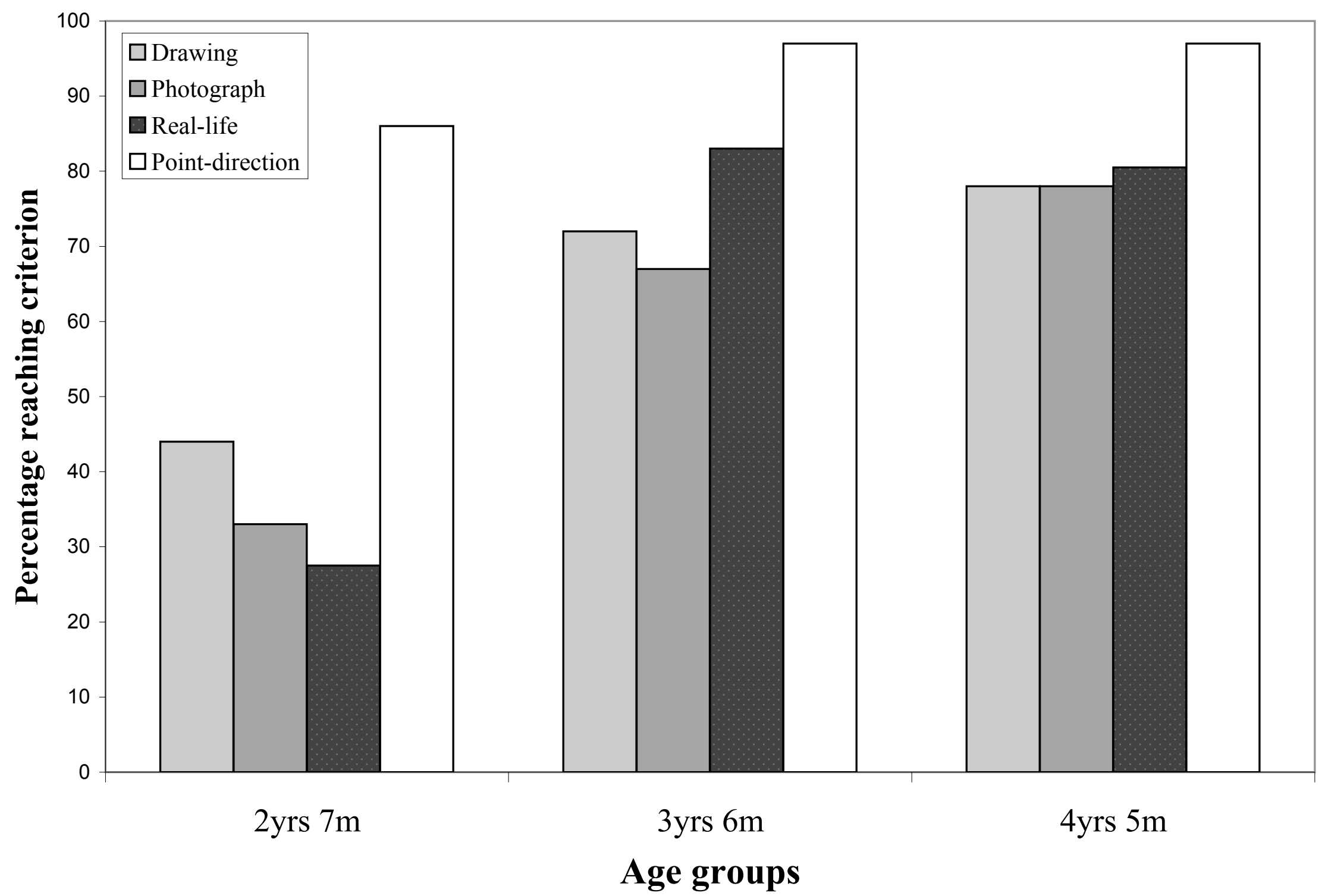




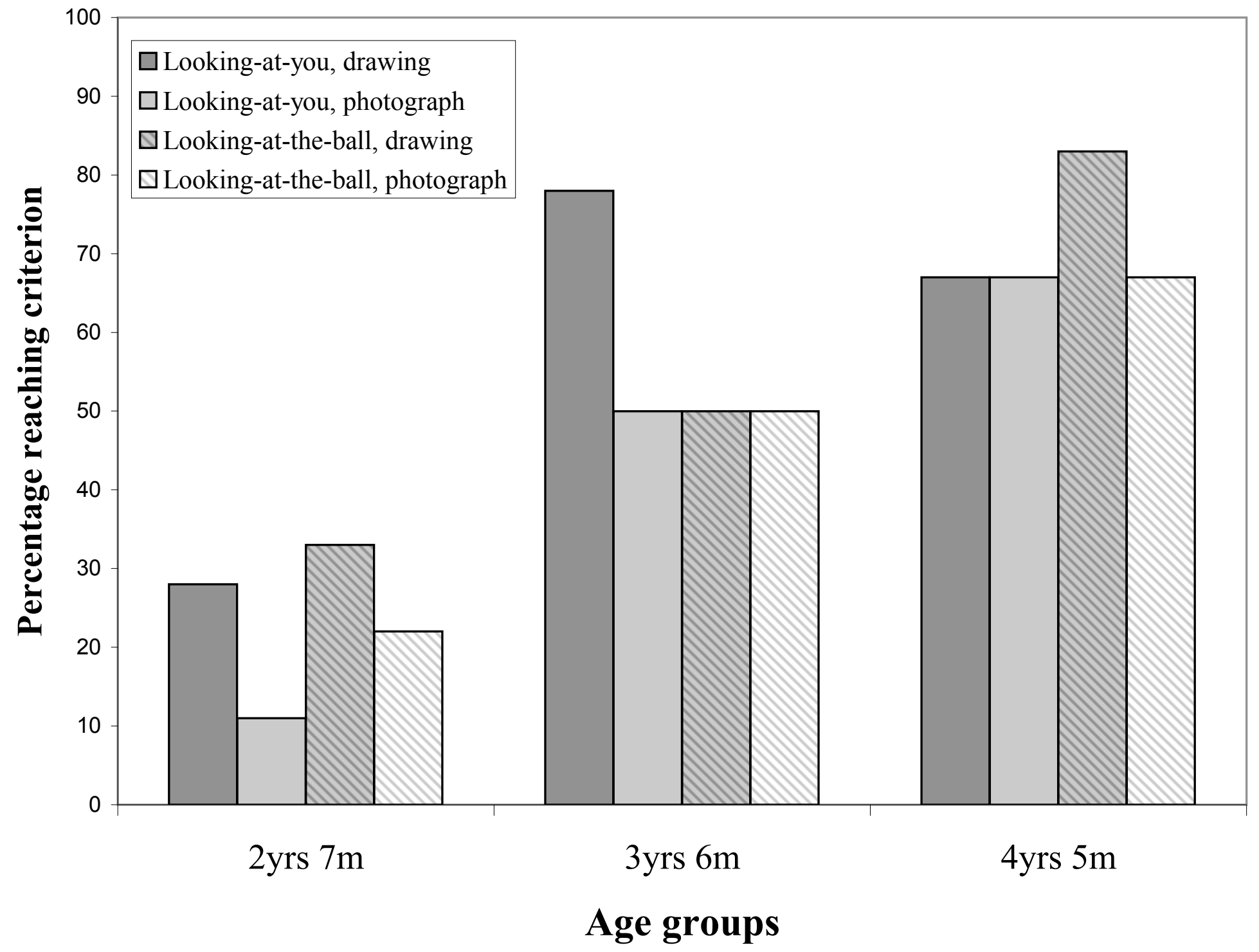

Age groups 


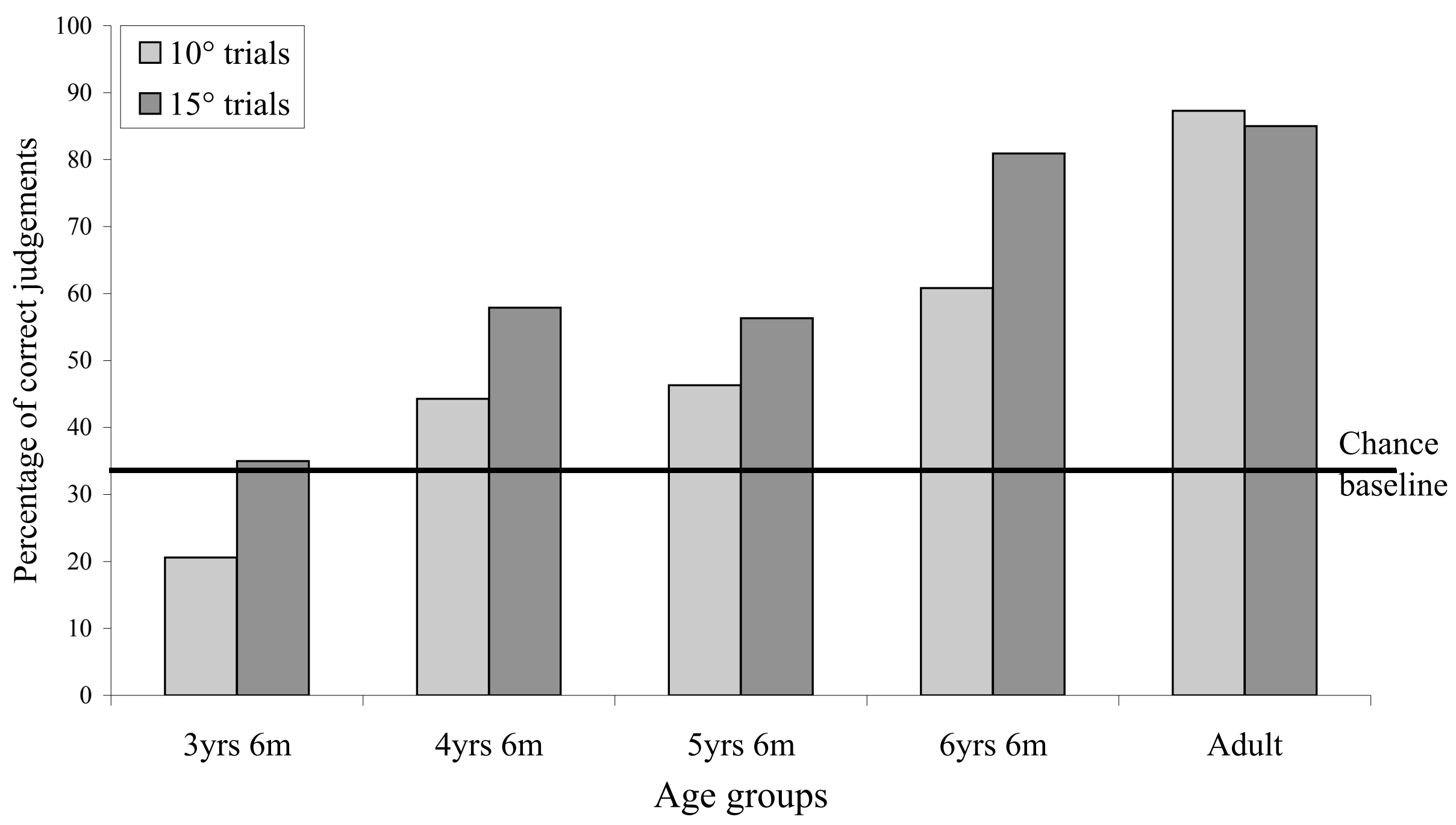




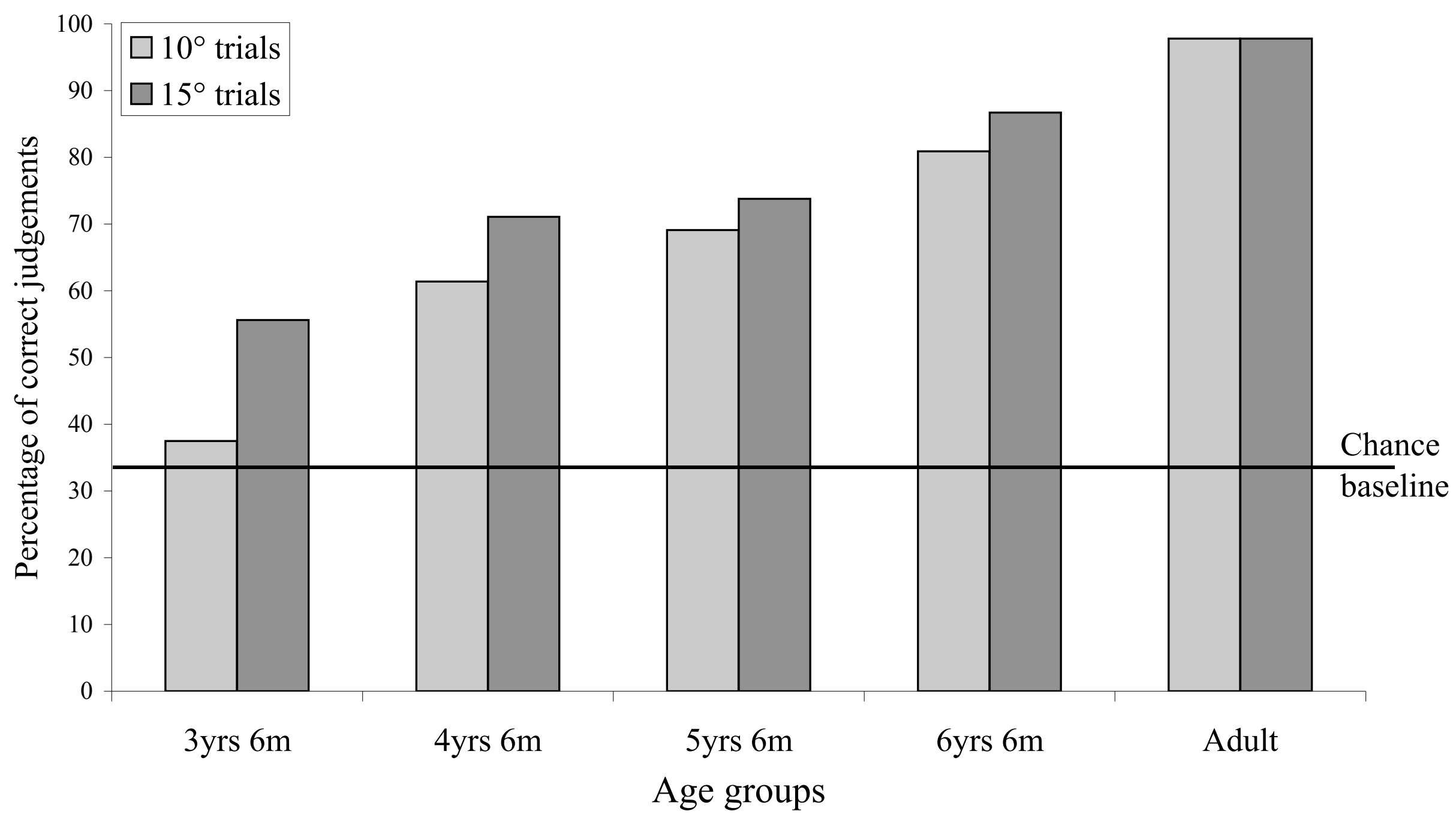




\section{Detailed response to the Editor's comments}

p. 14: Why not assess whether performance in each condition was significantly greater than expected by chance?

We decided to deal with the issue of success through chance responding by setting criteria, following previous research using these tasks. Viewed as a group, even the 2-year-olds performed significantly above chance on most tasks. Reporting the number of children reaching criterion at each age is therefore a more sensitive measure of developmental change, which was the main focus of the manuscript. However, we would be happy to report performance against chance if you feel it important.

p. 14, first complete paragraph: You state "a majority of 3- and 4-year-olds passed each task": This may be literally true, but on 3 of the 4 tasks, only 50\% of the 3-yearolds passed. Stating here (and on page 16) that "a majority" passed, seems to be overstating your data a bit

We have altered the wording in both sections to indicate that 3-year-old performance was only around $50 \%$

Also, you do not provide an examination of age differences. It appears from the figure that 4-year-olds performed significantly better than 3-year olds. This should be tested.

This analysis is now included. The 4-year-olds' performance was not significantly better than the 3-year-olds. The 3-year-olds performed significantly better than the 2year-olds on all but the drawing Looking-at-the-ball task.

p. 14, second complete paragraph: Provide data for these statements.

This has been provided.

p. 18, line 1: Replace "normal" with "typically developing"

This has been done.

p. 22, first paragraph: Provide marginal means, as well as the significance pattern for the main effect of age

These have been provided. 